\title{
Numerical Prediction of Wall Effect on Propeller in Restricted Channel
}

\author{
Zhen Ren, Decheng Wan* \\ State Key Laboratory of Ocean Engineering, School of Naval Architecture, Ocean and Civil Engineering, Shanghai Jiao Tong \\ University, Collaborative Innovation Center for Advanced Ship and Deep-Sea Exploration, Shanghai, China \\ Email: *dcwan@sjtu.edu.cn
}

How to cite this paper: Ren, Z. and Wan, D.C. (2019) Numerical Prediction of Wall Effect on Propeller in Restricted Channel. Journal of Applied Mathematics and Physics, 7, 1645-1659. https://doi.org/10.4236/jamp.2019.78112

Received: June 12, 2019

Accepted: August 9, 2019

Published: August 12, 2019

\begin{abstract}
In restricted channel, the hydrodynamic performance of propeller is affected by the wall. In the present work, two cylindrical channels with different diameters being 1.8D and 5.0D are adopted to study the influence of wall on the hydrodynamic performance and wake field of the propeller model DTMB4119. The numerical simulations are carried out by the single-phase solver pimpleDyMFoam in open source platform OpenFOAM. The Reynolds Averaged Navier-Stokes equations (RANS) are adopted to solve the flow field. The arbitrary mesh interface (AMI) method is used to simulate the rotation of propeller. The designed advance ratio, $J=0.833$, is applied in all the computations. For the $5.0 \mathrm{D}$ case, the predicted results of open water performance are in good agreement with experiment data. In restricted channel, the predicted results of thrust and torque coefficients are larger than the open water case. The pressure on the wall of restricted channel downstream increases and approaches the results in open water gradually. Due to the flux conservation, higher negative induced velocity is investigated in the flow field of the propeller in restricted channel.
\end{abstract}

\section{Keywords}

Wall Effect, Restricted Channel, Propeller, Hydrodynamic Performance

\section{Introduction}

When the ship advances in restricted water, the hydrodynamic characteristics of propeller will also be affected by the wall. It is necessary to study the hydrodynamic performance and flow mechanism of propeller in restricted water. The wall effect is the effect of wall in restricted water on the hydrodynamic performance of propeller. In practical applications, there are some special cases that 
propeller needs to be placed in restricted channel, such as bow thruster, ducted propeller and water tunnel. In restricted channel, the influence of surrounding wall on the hydrodynamic performance of propellers must be taken into consideration. The study on the wall effect can also provide suggestions for the design of bow thrusters and ducted propellers.

To study the wall effect of the restricted channel on the propeller, the extensive experiments were conducted. At the International Towing Tank Conference, scholars have repeatedly proposed the correcting methods for the wall effect on propeller in water tunnel. At the 10th International Towing Tank Conference in 1963 [1], the Wood Harris method was recommended to correct the wall effect and the correction coefficient curves were drawn. In their experiments, measurement points of velocity were placed in front of the propeller and at the disk. Huang and Chu [1] carried out the experiments of three geometrically similar propeller models in the water tunnel. They measured the velocity distribution at different cutting sections near the propeller disk and the pressure distribution on the wall of water tunnel. In their experiments, the Wood Harris method was adopted to correct the velocity. Qian and Chen [2] used the Wood Harris method to correct the advance coefficient and thrust of the ducted propeller in the model tests. The wall effect is also expressed as the blockage of propeller to the flow field. Catalina [3] studied the influence of blockage effect on the velocity distribution on the turbine blades in the water tunnel tests.

CFD is proved to be a robust and reliable tool to study the open water performance of propeller. And in this way, lot of data in flow field are provided, which is advantageous to analyze the mechanism of wall effect on the hydrodynamic performance of propeller. Huang, et al. [4] compared the effect of different water tunnel diameters on the hydrodynamic performance of propeller, DTMB 4119. But the pressure distribution on the blades was only compared without further analysis. Sorensen, et al. [5] presented a correction model for wall effect on rotors of wind turbines or propellers in wind tunnels and the validation was carried out by using RANS model coupling with actuator disc method. Chen, et al. [6] established an isotropic mixture multiphase model to study the wall effect that was based on RANS equations. Watanabe, et al. [7] analyzed the wall effect on the cavitation of propeller.

The bow thruster and ducted propeller are the typical applications of propeller in restricted channel. In the studies on the wall effect of bow thruster and ducted propeller, the focus is the effect of the tip clearance on the hydrodynamic performance of the propeller. Yongle, et al. [8] investigated the effects of different tip clearances on the performance of ducted propeller via numerical simulations at three different advance ratios. They found the clearance size has little effect on the pressure distribution on the blade below 0.98 spanwise. Based on the model experiments, Yan [9] used the commercial code, Fluent, to compute hydrodynamic performance of bow thruster. The MRF method was adopted to simulate the rotation of propeller. And three tip clearances were taken into consideration. $\mathrm{Yu}$, et al. [10] employed the panel method code, panMARE, and the RANSE 
code ANSYS-CFX to investigate the open water performance of Ka-series propeller with duct 19A. And the flow in three tip clearances was studied. Taking the ducted propeller JD75 $+\mathrm{Ka} 4-70$ as a research object, Lv, et al. [11] analyzed the wall effect of water tunnel with different diameters on the hydrodynamic performance. They found that if the ratio of the duct diameter to tunnel diameter is less than $1 / 3$, the predicted error of open water performance is within $5 \%$. It has been proved feasible to predict the hydrodynamic characteristics of propeller by using open source platform OpenFOAM. He, et al. [12] [13] [14] studied the open water performance of CRPs under different blade number ratio with pimple DyMFoam solver solving the flow field and arbitrary mesh interface (AMI) completing the rotation of propeller. Xu and Wan [15] [16] performed the numerical simulation of Propeller Boss Cap Fins by using pimple DyMFoam and analyzed the hydrodynamic characteristics, energy-saving mechanism and scale effect.

In the present studies on the wall effect, the focus is the wall effect on the hydrodynamic performance of propeller and the treatment of the blade tip clearance. And there are few studies on the flow mechanism in the flow field. To study the flow mechanism of the unsteady flow field of propeller in restricted channel, the numerical simulations of DTMB 4119 propeller in two water channels that the diameters are $1.8 \mathrm{D}$ and $5.0 \mathrm{D}$, respectively, are carried out. In the present simulations, RANS approach with the SST $k-\omega$ turbulence model is applied to calculate the flow field and pimple algorithm is employed to decouple the velocity and pressure.

This paper is organized as follows. The first part is the numerical method including the governing equations, turbulence model and arbitrary mesh interface. Then the pre-processing to generate the grids in the numerical simulation is presented. The results including the hydrodynamic performance, pressure on the blades and tunnel and wake filed are analyzed in detail. A brief conclusion is given at last.

\section{Numerical Method}

In the present work, DTMB 4119 propeller is used for all the simulations. The main parameters are presented in Table 1 . Figure 1 shows the geometry: left is $3 \mathrm{D}$ view, middle is front and right is side view, respectively.
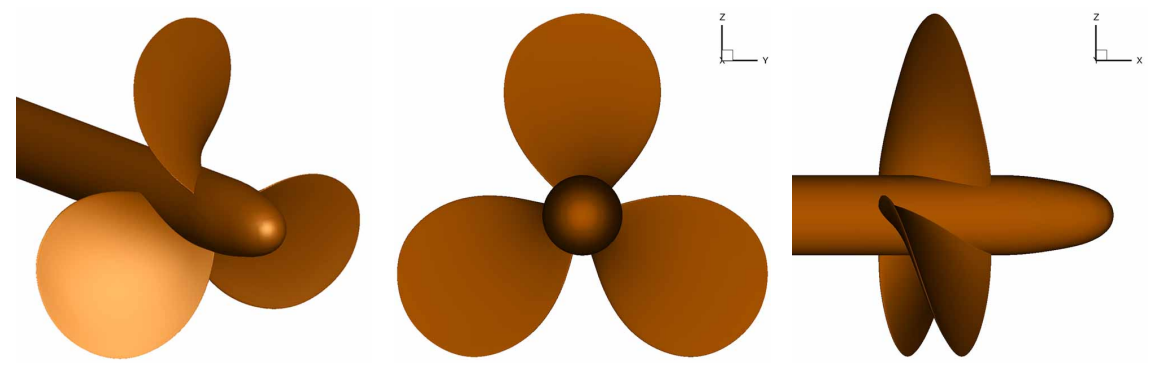

Figure 1. Geometry of DTMB 4119 propeller (left: 3D view; middle: front view; right: side view). 
Table 1. Parameters of propeller DTMB 4119.

\begin{tabular}{cccc}
\hline Parameters & Value & Parameters & Value \\
\hline $\mathrm{D}$ & $0.3048 \mathrm{~m}$ & Rake & $0^{\circ}$ \\
Pitch ratio (0.7 R) & 1.084 & Skew & $0^{\circ}$ \\
Number of blades & 3 & Section & NACA66mod \\
Hub ratio & 0.2 & Camber line & $\alpha=0.8$ \\
Rotation direction & Right & Expanded area ratio & 0.6 \\
\hline
\end{tabular}

\subsection{Governing Equations}

In the present simulations, the single-phase solver, pimpleDyMFoam in the open source platform OpenFOAM is adopted to calculate the flow field of propeller. The governing equations include the mass conservation Equation (1) and the momentum Equation (2), which can be written as:

$$
\begin{gathered}
\nabla \cdot U=0 \\
\rho \frac{D U}{D t}=-\nabla p+\left(\mu+\mu_{t}\right) \nabla^{2} U
\end{gathered}
$$

where $U$ is fluid velocity field; $p$ represents the pressure; $\rho$ is the mixture density; $\mu$ is dynamic viscosity, $\mu_{t}$ is turbulence viscosity.

\subsection{Turbulence Model}

For the turbulence model in the present numerical scheme, SST $k$ - $\omega$ model is selected because the turbulence model combines the advantages of the standard $k$ - $\varepsilon$ model and $k$ - $\omega$ model [17]. The turbulence model is able to ensure the accuracy and reliability of the solution at the wall. $k$ denotes turbulence kinetic energy and $\omega$ denotes dissipation rate. The transport equations of $k$ and $\omega$ are presented below:

$$
\begin{gathered}
\frac{\partial \rho k}{\partial t}+\nabla \cdot(\rho U k)=\nabla \cdot\left(\Gamma_{k} \nabla k\right)+\tilde{P}_{k}-D_{k} \\
\frac{\partial \rho \omega}{\partial t}+\nabla \cdot(\rho U \omega)=\nabla \cdot\left(\Gamma_{\omega} \nabla \omega\right)+P_{\omega}-D_{\omega}+Y_{\omega}
\end{gathered}
$$

where $\Gamma_{k}$ and $\Gamma_{\omega}$ are the effective diffusivity of $k$ and $\omega$, respectively. $\tilde{P}_{k}$ and $P_{\omega}$ are the turbulence production terms and $D_{k}$ and $D_{\omega}$ denote the turbulence dissipation terms. $Y_{\omega}$ represents the cross-diffusion term introduced by blending the standard $k-\omega$ and $k-\varepsilon$ models.

\subsection{Arbitrary Mesh Interface}

Traditionally, moving reference frame (MRF) is adopted in the steady prediction of propeller so that the flow filed obtained by this method is not real. Since the mechanism of the wall effect is to analyze, it is necessary to obtain a real unsteady flow field of the propeller. So the sliding mesh method is more suitable than MRF method in the simulations of propeller rotation. In the sliding mesh me- 
thod, the computational domain is divided into two regions. The two regions include a static region and a rotation region. Both regions exchange information via the overlapping area of interfaces, as shown in Figure 2. The blue mesh is the main interface in the rotation region while the red mesh is the slave interface in the static region. Although the grid size on the interface may be slightly different, the flux through the interface should be consistent in order to avoid the possible divergence in the calculations [18].

To analyze the wall effect in restricted channel, two conditions that the diameters of computational domain are 1.8 and 5.0 times diameter of propeller DTMB 4119 are set. The inlet is the constant velocity, advance speed $V_{A}$. The outlet is the second boundary condition. The cylinder surface is the non-slip wall. The surface of blades and shaft are the non-slip and generated wall. The boundary conditions of computational domain are shown in Figure 3.

\subsection{Mesh and Time Step}

In numerical simulations, mesh and time step are critical to the predictions. So the mesh sensitivity study and time step check are necessary. In the present study, three grid schemes for case D1.8 are used to investigate the influence of grid size on the numerical predictions. As can be seen in Table 2, all hex-unstructured grids are generated with different resolution level. To capture

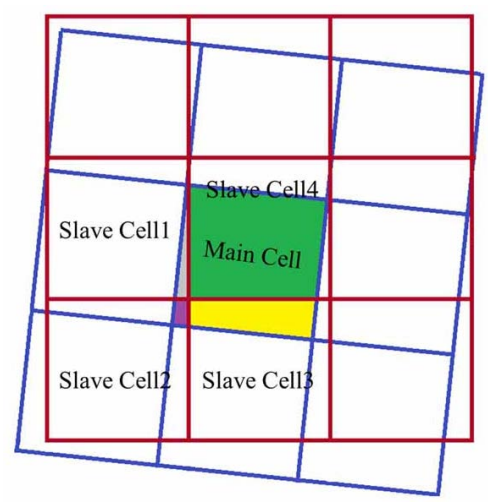

Figure 2. Arbitrary mesh interface.

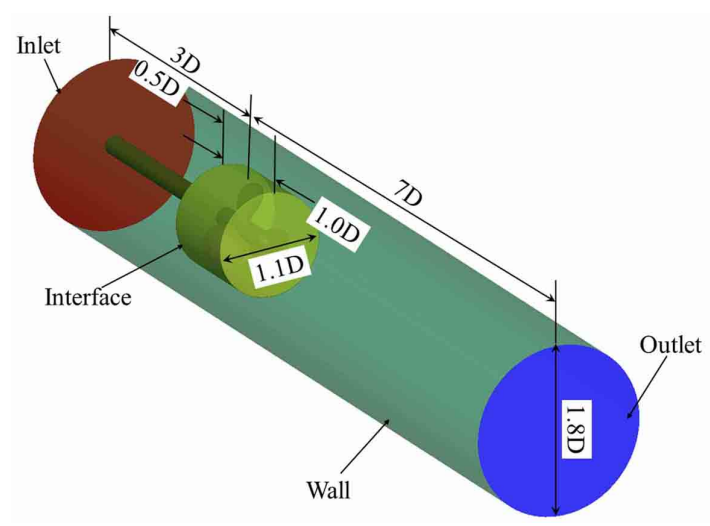

Figure 3. Domain and boundary conditions. 
Table 2. Grids number with different resolution level.

\begin{tabular}{cccc}
\hline Grid & Nodes at rotating & Nodes at static & \multicolumn{1}{c}{ Total } \\
\hline Coarse & 487,329 & 393,016 & 880,345 \\
Mid & 886,522 & 689,820 & $1,576,342$ \\
Fine & $1,713,044$ & $1,346,548$ & $3,059,592$ \\
\hline
\end{tabular}

the blades well, the surface mesh size of blades to $D$ is $0.2 \%$ while the edges needs to be refined so that the size to $D$ is $0.1 \%$. Near the wall of blades, the prism layers are added with $y+$ being 40 . The surface mesh of blades is presented in Figure 4.

In the unsteady simulations, the choice of time steps has a large impact on the simulation results. To check the influence of time step on the numerical prediction, three time steps that are $2.778 \mathrm{e}-4,5.556 \mathrm{e}-4,1.111 \mathrm{e}-3$, during which the propeller rotates by $1^{\circ}, 2^{\circ}$, and $4^{\circ}$ respectively, are selected.

\section{Results and Analysis}

\subsection{Hydrodynamic Performance}

To study the mesh sensitivity in the numerical predictions, the simulations of DTMB 4119 at $J=0.833$ are performed with the rotational speed of propeller being $600 \mathrm{rpm}$. The predicted results of thrust and torque coefficients obtained by three grid schemes are compared with the experiment data [19] and the relative errors are also listed in Table 3 . The calculated values of hydrodynamic coefficients of the propeller obtained from the medium resolution mesh are approaching to those on the meshes with fine resolution. Although the predicted results with fine resolution are more accuracy, the calculation is more time-consuming. So in the present study, the medium resolution level mesh is adopted to perform the next simulations.

Figure 5 depicts the time histories of thrust coefficient with different time step schemes. The predicted results of thrust coefficient $K_{t}$ with minimum and medium time step are almost the same. And the tendency of torque coefficient $10 K_{q}$ is the same as the thrust coefficient. Taking the time-consuming into consideration, the medium time step is used.

Figure 6 shows the time histories of thrust and torque coefficients of the propeller at $J=0.833$ for both cases. As we can see, the two curves, thrust and torque coefficients, have converged and remained stable after $0.2 \mathrm{~s}$. The thrust of the propeller in restricted channel is always larger than that in the open water, and the tendency of torque is the same as the thrust. This is because that in order to maintain flux conservation, the advance speed of the propeller in restricted channel is relatively smaller than the open water case.

Table 4 presents the comparison between the predicted results and experimental data. In open water, the predicted results are in good agreement with the experimental data, with the error of thrust and torque coefficients are less than 

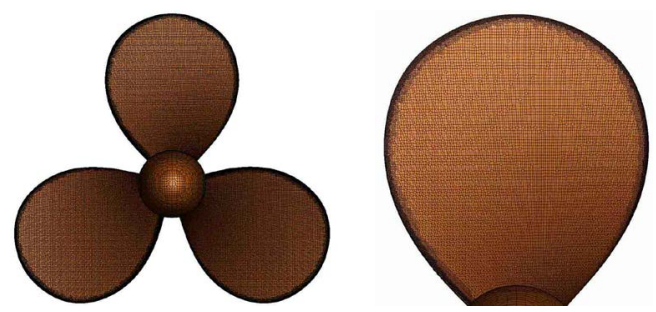

Figure 4. Distribution of grids (left: propeller grid; right: blade grid).

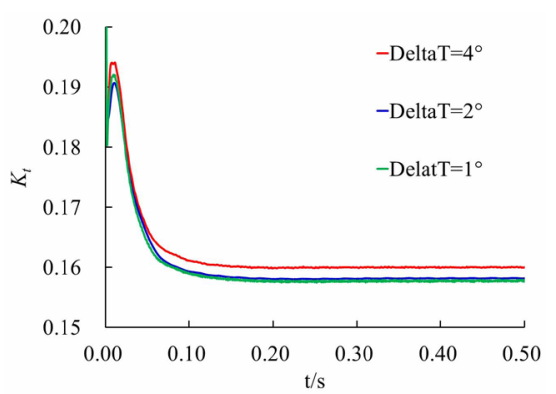

Figure 5. Time histories of $K_{t}$ with different time step schemes.

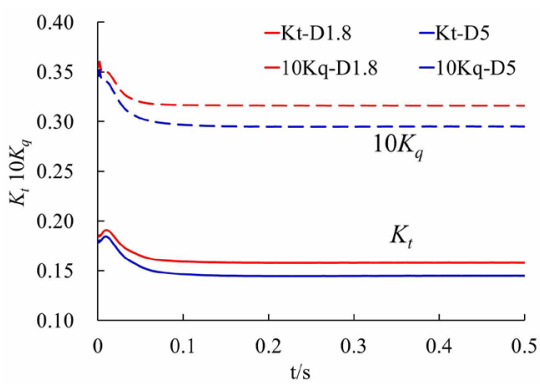

Figure 6. Time histories of thrust and torque coefficient at $J=0.833$.

Table 3. Computational results with different mesh schemes.

\begin{tabular}{ccccc}
\hline & $K_{t}$ & Error & $10 K_{q}$ & Error \\
\hline EFD & 0.1460 & & 0.280 & \\
Coarse & 0.1604 & $9.84 \%$ & 0.3350 & $19.64 \%$ \\
Mid & 0.1585 & $8.54 \%$ & 0.3213 & $14.74 \%$ \\
Fine & 0.1582 & $8.38 \%$ & 0.3160 & $12.84 \%$ \\
\hline
\end{tabular}

Table 4. Comparison of predicted and experimental data [19].

\begin{tabular}{cccc}
\hline \multirow{2}{*}{ Parameter } & EFD & \multicolumn{2}{c}{ CFD } \\
\cline { 3 - 4 } & & D1.8 & Error \\
\hline$K_{t}$ & 0.146 & 0.1585 & $8.54 \%$ \\
$10 K_{q}$ & 0.280 & 0.3213 & $14.74 \%$ \\
& & D5.0 & Error \\
$K_{t}$ & 0.146 & 0.1449 & $-0.76 \%$ \\
$10 K_{q}$ & 0.280 & 0.2951 & $5.41 \%$ \\
\hline
\end{tabular}


$-1.0 \%$ and $6.0 \%$, respectively. The thrust of the propeller in restricted channel increases by approximately $9.0 \%$ compared to the open water condition. Meanwhile, the torque increases by more than $9.0 \%$.

\subsection{Pressure on Blades}

Figure 7 depicts the pressure distribution on the blades. The top is the results in restricted channel, case D1.8. The bottom is the result in the open water, case D5.0. Left is the pressure side and right is the suction side. When comparing the pressure distribution on the pressure side, it is found that the pressure near the leading edge in restricted channel is larger than the open water. While, the pressure near the hub in restricted channel is less than that in the open water. On the suction sides, the area of the low pressure region in restricted channel is larger than another case. Larger area of low pressure causes a slight increase of thrust and torque of propeller in restricted channel.

\subsection{Pressure on Tunnel Wall}

The pressure on the wall varies downstream due to the propeller rotation. Figure 8 presents the variation of pressure coefficient on the wall of the tunnel downstream. For the case D5.0, the pressure coefficient remains essentially constant along the circumference, while three peaks occur in restricted channel because the propeller DTMB 4119 owns 3 blades. In front of the propeller disk, $x / D=-0.30$ and -0.20 , the pressure relatively increases when the position is closer to the disk. For the case D1.8, the pressure on the wall downstream gradually
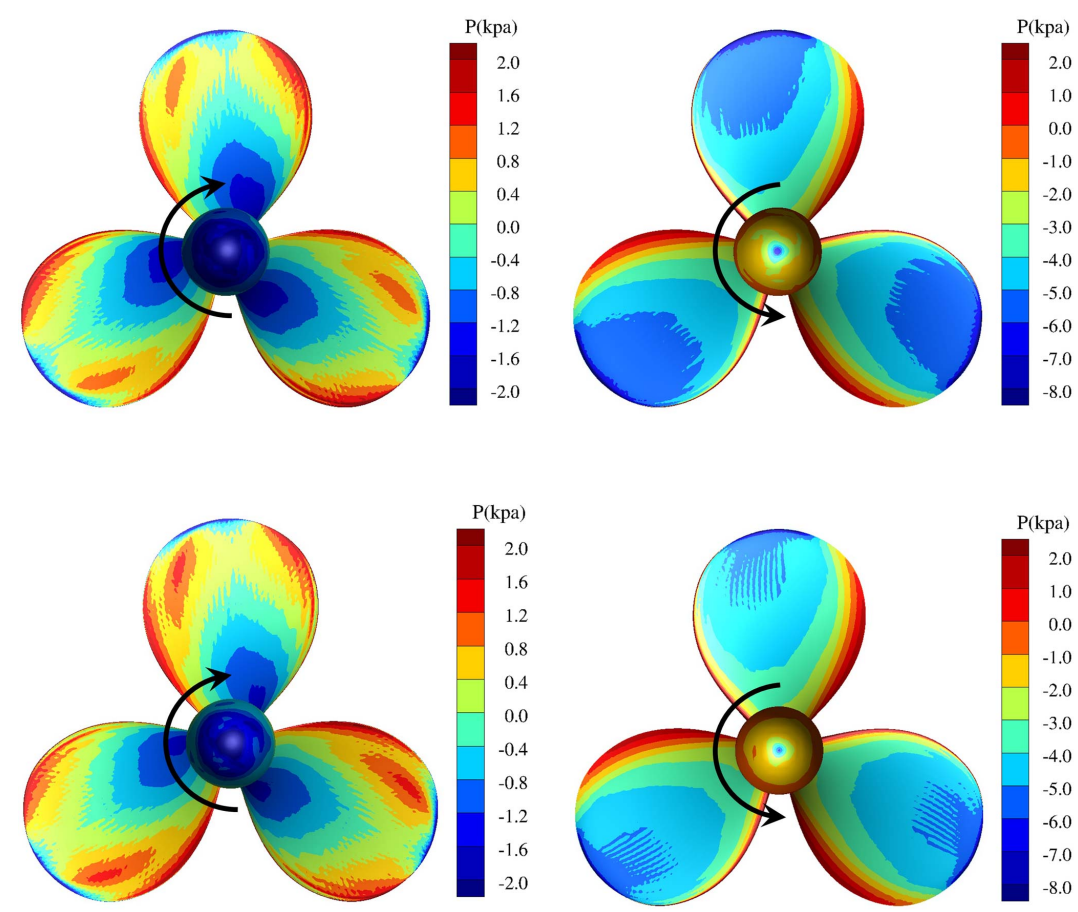

Figure 7. Pressure distribution on blades (top: restricted channel; bottom: open water). 


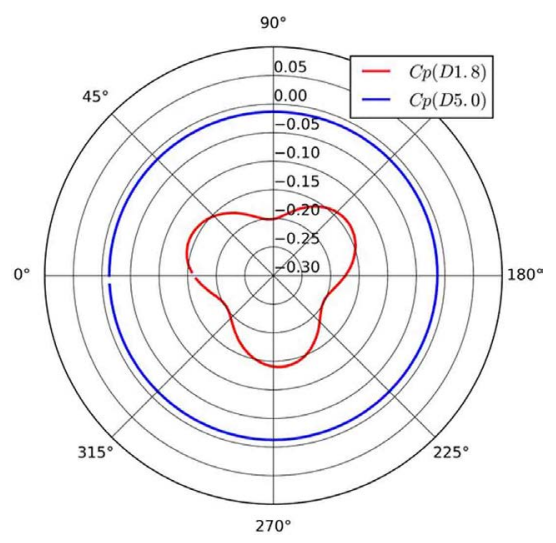

$x / \mathrm{D}=-0.30$

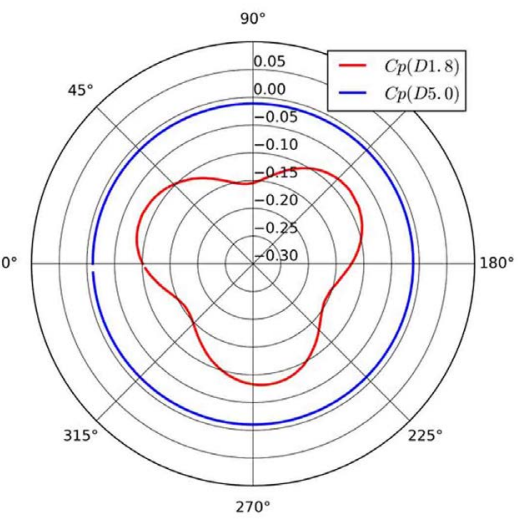

$x / \mathrm{D}=0.0$

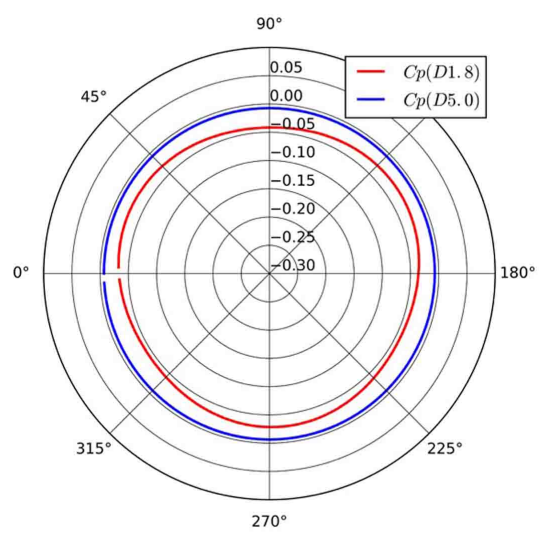

$x / D=0.50$

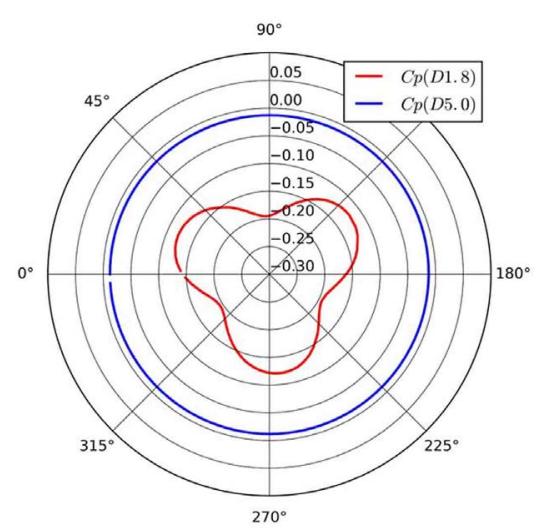

$x / \mathrm{D}=-0.20$

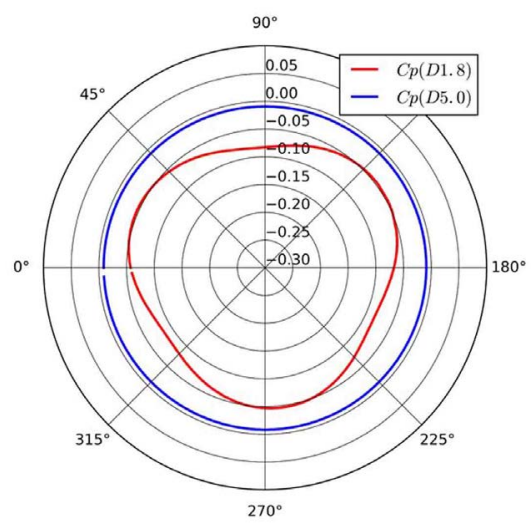

$x / \mathrm{D}=0.25$

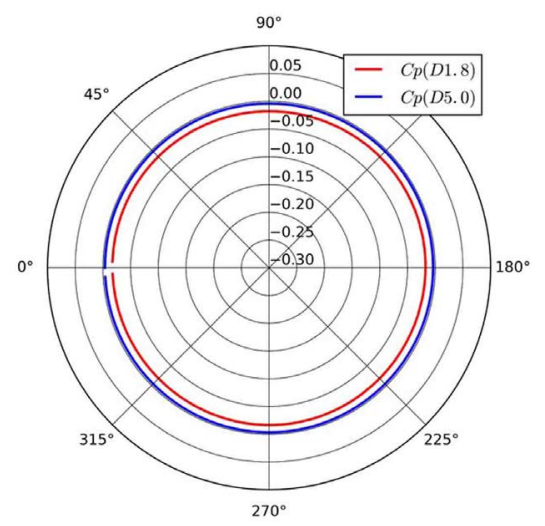

$x / D=1.00$

Figure 8. Pressure coefficient distribution on the wall of tunnel (Red: restricted channel; Blue: open water).

increases and approaches the results under the open water condition. In restricted channel, the decreasing pressure peaks indicates that the influence of the propeller rotation on the wake field is weakening gradually downstream. During the entire development downstream, the pressure coefficient under open water conditions increases slightly, the value is approximately zero at $x / D=1.0$, but remains constant in the circumference basically. 


\subsection{Wake Field}

The variation of the pressure on the tunnel wall is closely related to the velocity distribution in the flow field. The axial velocity distribution is presented in Figure 9. In the slipstream region where $\mathrm{z} / \mathrm{D}$ is from -0.5 to 0.5 approximately, the axial velocity in open water is larger than the results in restricted channel, especially in the far field. Under both conditions, the radius of slipstream region is constant downstream basically and maintain around the radius of propeller. In restricted channel, the axial velocity outside the slipstream region is much less than the results in open water.

The variation of the pressure coefficient on the wall is caused by the difference of the velocity in the flow field. Figure 10 shows the axial velocity in the Cartesian coordinate system. Left are the results in restricted channel with right being obtained in open water. The black curve in the figure indicates the position of $1.8 \mathrm{D}$.

Corresponding to the position of the three blades of the propeller DTMB 4119 , there are three high-speed regions in the inflow before the propeller disk, $x / D=-0.30$. Obviously, the axial velocity in restricted channel is less than that in the open water. The maximum of axial velocity in open water is larger than 1.05 while the greatest value in restricted channel is between 1.04 and 1.05. The lower advance speed results in the larger thrust and torque, as shown in Figure 6. And on the other hand, the area of the region accelerated by the propeller in the open water is larger than that in restricted channel.

At the propeller disk, $x / D=0.0$, the difference in axial velocity distribution is mainly concentrated in the peripheral region of the diameter of the propeller, where the velocity in restricted channel is less than the case D5.0. For both cases, the slipstream region is presented clearly downstream at $x / D=0.25$ and 1.0. In the slipstream region, the flow is significantly accelerated and a distinct boundary is shown compared with the surrounding area. As shown in Figure 10(d), the axial velocity outside the slipstream in restricted channel region is less than that in the open water. While the axial velocity in the slipstream region in open water is much larger than another case.
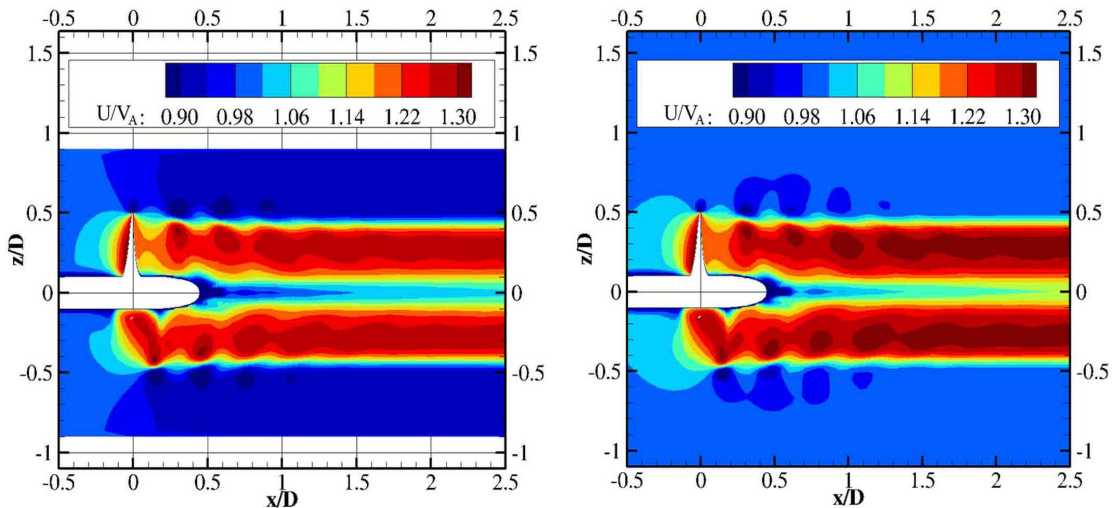

Figure 9. Axial velocity distribution (left: restricted channel; right: open water). 

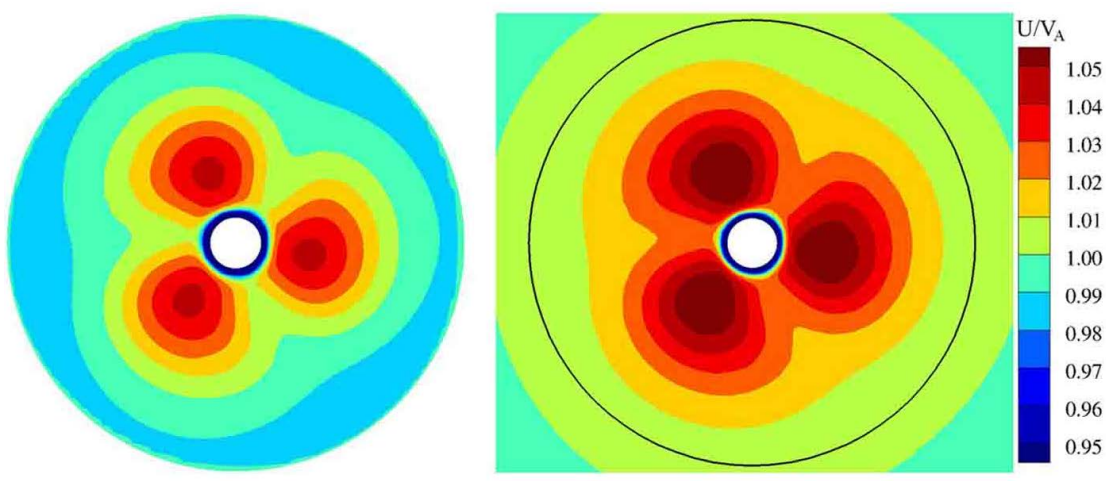

(a)
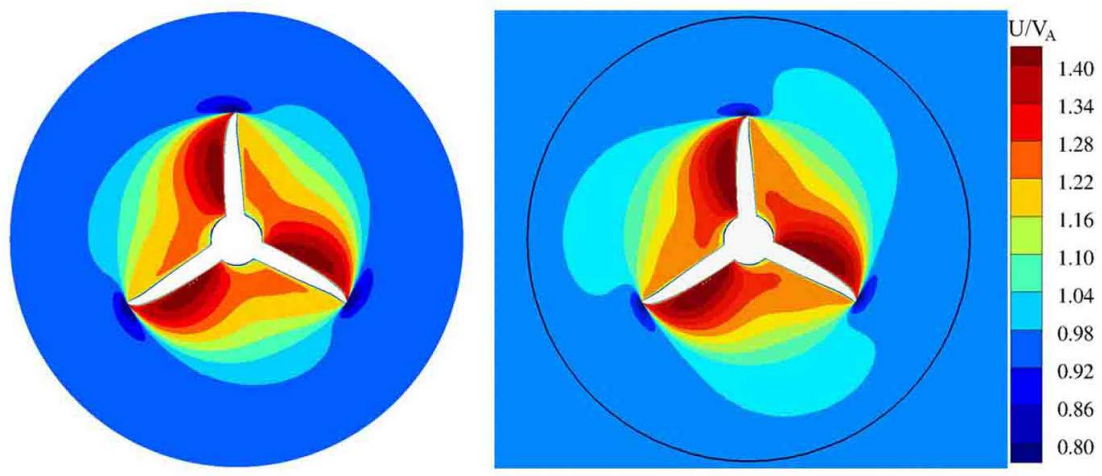

(b)
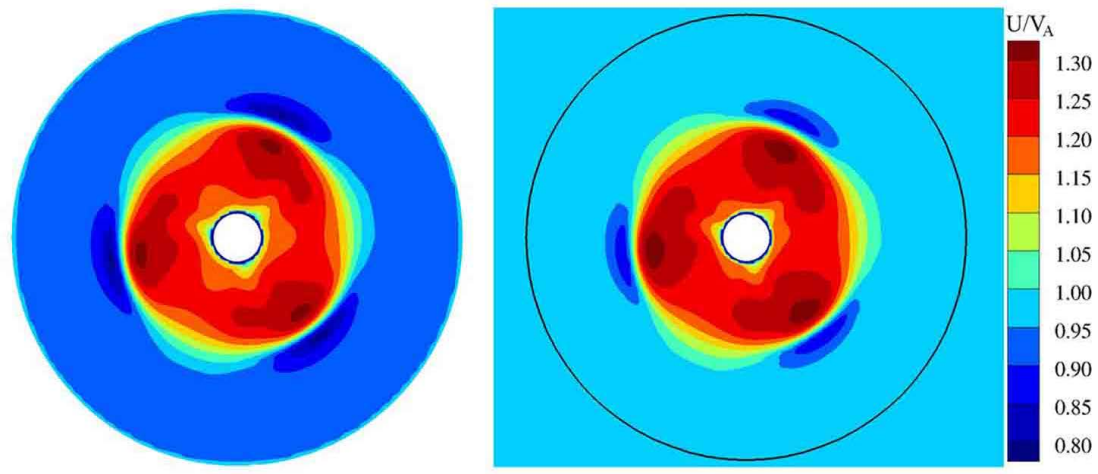

(c)
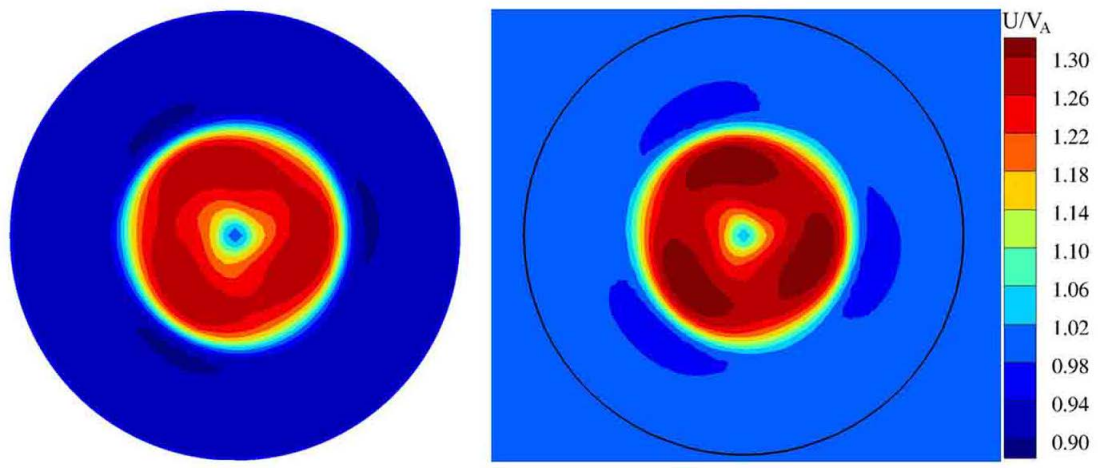

(d)

Figure 10. Axial velocity at different cutting planes (a: $x / D=-0.30 ; \mathrm{b}: x / D=0.0 ; \mathrm{c}: x / D=$ $0.25 ; \mathrm{d}: x / D=1.0$; left: restricted channel; right: open water). 
Figure 11 shows the hydrodynamic model of ideal thruster. $\mathrm{AA}_{1}, \mathrm{BB}_{1}$ and $\mathrm{CC}_{1}$ are the position of the far field upstream, propeller disk and far field downstream. $r$ represents the radius of the propeller. The solid curves represent the boundary of slipstream region. When a thruster operates in restricted channel, the boundary, $A B C$ and $A_{1} B_{1} C_{1}$, is the wall that is not penetrated. Since the fluxes through the sections, $\mathrm{AA}_{1}$ and $\mathrm{CC}_{1}$, are conserved per unit time, the following formula can be obtained:

$$
\begin{gathered}
V_{A} \cdot 2 R=\left(V_{A}+u_{a}\right) \cdot 2 r_{1}+\left(V_{A}+u_{a 1}\right) \cdot 2\left(R-r_{1}\right) \\
u_{a 1}=\frac{r_{1}}{r_{1}-R} u_{a}
\end{gathered}
$$

where, $V_{A}$ is the advance speed, $R$ is the radius of restricted channel, $r_{1}$ represents the radius of slipstream region that is the radius of propeller substantially. $u_{a}$ is the axial induced velocity in the slipstream region while $u_{a 1}$ is the axial induced velocity outside the slipstream region.

Equation (5) expresses the flux conservation in the water tunnel. In the slipstream region, the flow is accelerated leading to an always positive axial induced velocity, $u_{a}$. The radius of slipstream region, $r_{1}$, that is approximately constant and equal to the radius of propeller, is less than the restricted channel. As expressed in Equation (6), the axial induced velocity outside the slipstream region, $u_{a 1}$, is negative and when $R$ approaches $r_{1}$ gradually, the negative induced velocity increases. So that the advance speed in restricted channel is less than that in open water.

In order to verify the theoretical model, the vector distribution of axial induced velocity in the flow field is shown in Figure 12. As we can see, negative induced velocity, $u_{a 1}$, occurs in both conditions. Due to the rotation of the propeller, the inflow before the propeller disk is accelerated and the wake field is accelerated significantly in the slipstream region where the axial induced velocity, $u_{a}$, reaches to 0.30 times or more advance speed. In order to maintain the flux conservation, negative induced velocity occurs outside the slipstream region downstream. It corresponds to the theoretical conclusion that the value of negative induced velocity in restricted channel is larger than that in open water. In restricted channel, the negative induced velocity approaches 0.10 time advance speed so that the total axial velocity is less than 1.0 and about 0.9 as shown in

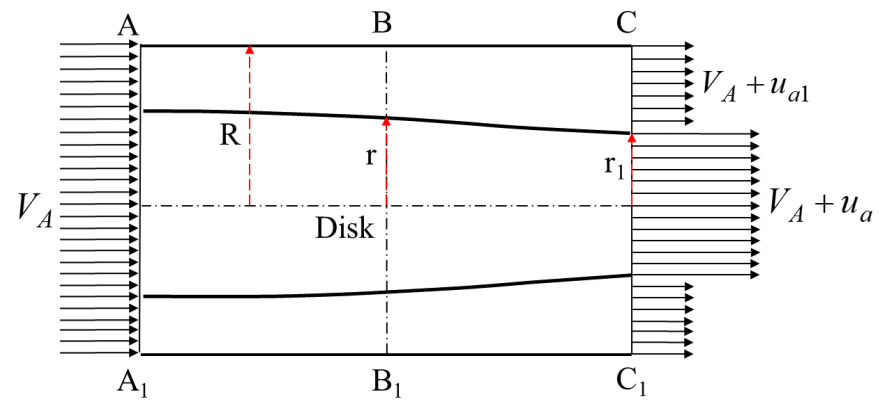

Figure 11. Hydrodynamic model of ideal thruster. 

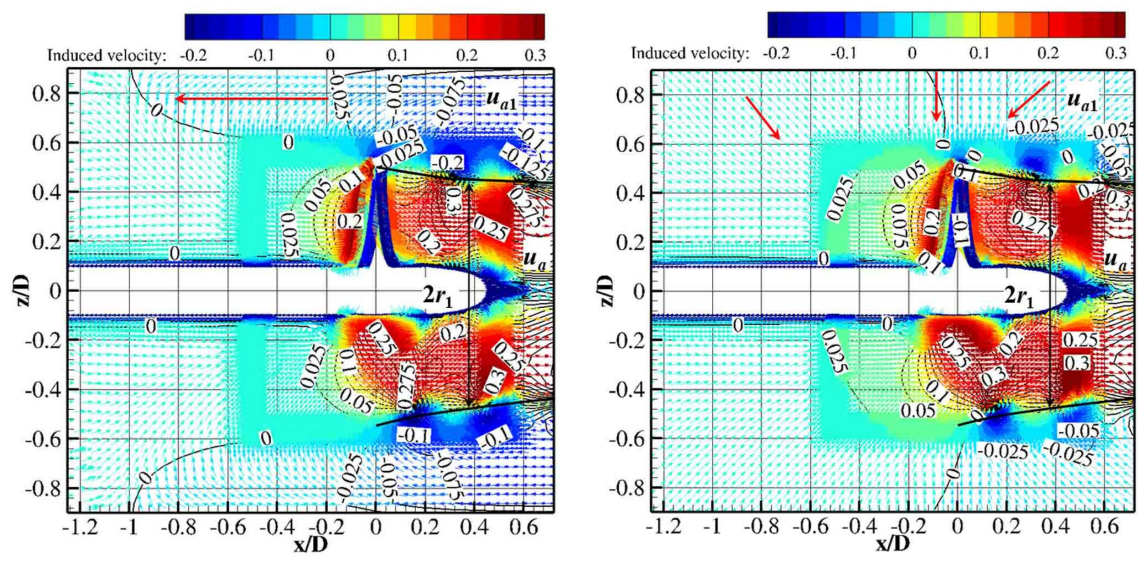

Figure 12. Induced velocity vector distribution (Left: restricted channel; right: open water).

Figure 9. The positive and negative induced velocity meet near $x / D=-1.0$ upstream, causing the inflow velocity of the propeller to decrease relatively. While in open water, the negative induced velocity is very little since the region outside the slipstream region is larger than that in restricted channel. And under this condition, the axial induced velocity flow into the disk from all the sides.

\section{Conclusions}

In the present work, the hydrodynamic performance of DTMB 4119 propeller under open water and restricted channel are predicted by the single-phase solver, pimple DyMFoam in the open source platform OpenFOAM. In restricted channel, the thrust and torque of propeller increase slightly compared with the results in open water. The pressure on the wall gradually increases and approaches the results under the open water condition with the influence of propeller on the wake field weakening. To maintain the flux conservation, the negative induced velocity occurs in restricted channel. When the radius of the channel approaches to the radius of the propeller, the negative induced velocity increases. The advance speed is reduced relatively, resulting in the higher thrust and torque.

Numerical simulations are only carried out at the design advance ratio. In the future, numerical simulations at the full speed should be carried out to analyze the hydrodynamic performance and mechanism of the propeller in the restricted waters at higher and lower advance speeds. In addition, smaller channel diameters should be studied since the gap between the propeller and the channel is very small in practical applications.

\section{Acknowledgements}

This work is supported by the National Natural Science Foundation of China (51879159, 51490675, 11432009, 51579145), Chang Jiang Scholars Program (T2014099), Shanghai Excellent Academic Leaders Program (17XD1402300), Program for Professor of Special Appointment (Eastern Scholar) at Shanghai Insti- 
tutions of Higher Learning (2013022), Innovative Special Project of Numerical Tank of Ministry of Industry and Information Technology of China (2016-23/09) and Lloyd's Register Foundation for doctoral student, to which the authors are most grateful.

\section{Conflicts of Interest}

The authors declare no conflicts of interest regarding the publication of this paper.

\section{References}

[1] Huang, P.T. and Chu, Y.Q. (1984) The Wall Effect and Correction of Cavitation Tunnel on Propeller Model Tests. Shipbuilding of China, 3, 3-12.

[2] Qian, X.N. and Chen, L.X. (1983) The Wall Effect of Cavitation Tunnel on Propeller Model Test. Shipbuilding of China, 3, 16-24.

[3] Lartiga, C. (2012) Development of a Rig and Testing Procedures for the Experimental Investigation Of Horizontal Axis Kinetic Turbines. Master Thesis, University of Victoria, Victoria.

[4] Huang, G.Y., Li, L.W., Zhao, Y. and Ye, H.K. (2011) The Wall Effect of Simple Propeller Water Tank by CFD. Ship Science and Technology, 33, 19-23.

[5] Sorensen, J.N., Shen, W.Z. and Mikkelsen, R. (2006) Wall Correction Model for Wind Tunnels with Open Test Section. AIAA Journal, 44, 1890-1894. https://doi.org/10.2514/1.15656

[6] Chen, X., Lu, C.J., Li, J. and Pan, Z.C. (2008) The Wall Effect on Ventilated Cavitating Flows in Closed Cavitation Tunnels. Journal of Hydrodynamics, 20, 561-566. https://doi.org/10.1016/S1001-6058(08)60095-5

[7] Watanabe, S. and Brennen, C.E. (2003) Dynamics of a Cavitating Propeller in a Water Tunnel. Journal of Fluids Engineering, 125, 283-292. https://doi.org/10.1115/1.1524588

[8] Yongle, D., Baowei, S. and Peng, W. (2015) Numerical Investigation of Tip Clearance Effects on the Performance of Ducted Propeller. International Journal of Naval Architecture and Ocean Engineering, 7, 795-804.

https://doi.org/10.1515/ijnaoe-2015-0056

[9] Yan, Z.G. (2012) Based on CFD Method to Research the Hydrodynamic Performance of Transverse Thrusters. Master Thesis, Jiangsu University of Science and Technology, Zhenjiang.

[10] Yu, L., Greve, M., Druckenbrod, M. and Abdel-Maksoud, M. (2013) Numerical Analysis of Ducted Propeller Performance under Open Water Test Condition. Journal of Marine Science and Technology, 18, 381-394. https://doi.org/10.1007/s00773-013-0215-4

[11] Lv, X.J., Zhou, Q.D. and Pan, Y.C. (2017) Wall Effect on Ducted Propellers in Water Tunnel Test. Journal of Naval University of Engineering, 29, 62-66.

[12] He, D.Y. and Wan, D.C. (2017) Numerical Investigation of Open Water Performance of CRPs under Different Blade Number Ratio. Proceedings of 14th National Conference of Hydrodynamics, Changchun, 8-13 August 2017, 1005-1012.

[13] Zheng, J., He, D.Y. and Wan, D.C. (2018) Numerical Analysis of Different Blade Number Ratios Influence on Open Water Performance of CRPs Based on CFD Method. Chinese Journal of Hydrodynamics, 33, 169-175. 
[14] He, D.Y. and Wan, D.C. (2018) Investigation of Hydrodynamic Performance of Contro-Rotating Propellers with Different Design Parameters. The Ocean Engineering, 36, 19-29.

[15] Xu, L.R. and Wan, D.C. (2018) Numerical Research on Hydrodynamic Characteristics of Propeller Boss Cap Fins. Chinese Journal of Ship Research, 13, 15-21.

[16] Xu, L.R. and Wan, D.C. (2018) CFD Investigation on the Energy-Saving Mechanism of Propeller Boss Cap Fins. Chinese Journal of Hydrodynamics, 33, 428-434.

[17] Menter, F.R., Kuntz, M. and Langtry, R. (2003) Ten Years of Industrial Experience with the SST Turbulence Model. Turbulence, Heat and Mass Transfer, 4, 625-632.

[18] Xu, L.R. and Wan, D.C. (2018) Numerical Investigation of Scale Effect for Propeller Boss Cap Fins. 28th International Ocean and Polar Engineering Conference, Sapporo, 10-15 June 2018, 805-811.

[19] Jessup, S.D. (1989) An Experimental Investigation of Viscous Aspects of Propeller. Ph.D. Thesis, Catholic University of America, Washington. 